\title{
THE FOURIER TRANSFORM IS ONTO ONLY WHEN THE GROUP IS FINITE
}

\author{
COLIN C. GRAHAM ${ }^{1}$
}

ABSTRACt. A very simple proof of the result of the title is given. Unlike previous proofs, the one presented here uses no results of harmonic analysis beyond the Pontryagin duality theorem.

THEOREM. Let $G$ be a LCA group with dual $\hat{G}$. If the Fourier transform

$$
\mathscr{F}: L^{1}(G) \rightarrow C_{0}(\hat{G})
$$

is onto, then $G$ is finite.

This result was proved in [2], [3] and more recently by Friedberg [1]. We give an even simpler proof than those of [1], [2], [3]. I am grateful to the referee for calling my attention to [4], which contains a proof of the corresponding result for some noncommutative groups.

Proof. From the Pontryagin duality theorem and the closed graph theorem, we know $\mathscr{F}$ is an isomorphism of Banach spaces, so $\mathscr{F}^{*}$ will also be an isomorphism of the corresponding dual spaces $\mathscr{F}^{*}: M(\hat{G}) \rightarrow$ $L^{\infty}(G)$. A simple computation shows that if $\mu \in M(G)$ then $\mathscr{F} * \mu(x)=$ $\hat{\mu}(x)$ almost everywhere, where $\hat{\mu}$ is the Fourier-Stieltjes transform of $\mu$. But if $G$ is not discrete, and $U \subseteq G$ is an opening, relatively compact nondense set, then the characteristic function of $U$ is not equal almost everywhere to a continuous function. Since each $\hat{\mu}(\mu \in M(\hat{G}))$ is continuous, $G$ can only be discrete and $\mathscr{F} * \mu=\hat{\mu}$ everywhere.

We consider $\mathscr{F}^{*}$ restricted to $L^{1}(\hat{G})$. Because $\mathscr{F} *$ is an isomorphism of Banach spaces, $\mathscr{F}^{*} L^{1}(\hat{G})$ is uniformly closed in $L^{\infty}(\hat{G})$. Of course, $\mathscr{F} * L^{1}(\hat{G})$ is also dense in $C_{0}(G)$. Thus $\mathscr{F}^{*}: L^{1}(\hat{G}) \rightarrow C_{0}(G)$ is onto. By the argument of the first paragraph, $\hat{G}$ is discrete. Thus $G$ is discrete and compact so $G$ is finite. Q.E.D.

\section{REFERENCES}

1. S. H. Friedberg, The Fourier transform is onto only when the group is finite, Proc. Amer. Math. Soc. 27 (1971), 421-422.

Received by the editors February 28, 1971 and, in revised form, July 19, 1972.

AMS (MOS) subject classifications (1970). Primary 42A68, 43A25; Secondary $46 \mathrm{~J} 10$.

${ }^{1}$ Partially supported by National Science Foundation Grant GP-32116.

(c) American Mathematical Society 1973 
2. M. Rajagopalan, Fourier transform in locally compact groups, Acta Sci. Math. (Szeged) 25 (1964), 86-89. MR 29 \#6250.

3. I. E. Segal, The class of functions which are absolutely convergent Fourier transforms, Acta Sci. Math. (Szeged) 12 (1950), Pars B, 157-161. MR 12, 188; 1002.

4. G. Rabson, The existence of nonabsolutely convergent Fourier series on compact groups, Proc. Amer. Math. Soc. 10 (1959), 893-897. MR 22 \#2910.

Department of Mathematics, Northwestern University, Evanston, Illinois 60201 